\title{
BARRERAS Y FACILITADORES A LA SUPLEMENTACIÓN CON MICRONUTRIENTES EN POLVO. PERCEPCIONES MATERNAS Y DINÁMICA DE LOS SERVICIOS DE SALUD
}

\author{
Juan Pablo Aparco',a, Lucio Huamán-Espino ${ }^{1, b}$
}

\begin{abstract}
RESUMEN
Objetivos. Explorar las barreras y facilitadores para la suplementación con micronutrientes en polvo (MNP) en madres de niños de 6 a 35 meses de edad de Tacna, Loreto, Puno y Lima. Materiales y métodos. Estudio cualitativo, exploratorio con enfoque de teoría fundamentada, realizado en Lima, Tacna, Loreto y Puno en el año 2014. Se aplicaron entrevistas a profundidad a madres de niños menores de tres años y observación en establecimientos de salud (ES) para explorar las barreras y facilitadores a la suplementación con MNP. Resultados. Se encontró como barreras: a nivel de sistema de salud, a las dificultades para acceder al ES, los rumores negativos al suplemento en la sala de espera y el maltrato del personal de salud; a nivel del producto (MNP), la falta de conocimiento sobre el suplemento, el sabor y los efectos secundarios y a nivel de la madre, aparecen barreras como la falta de tiempo, el olvido, las dificultades en la preparación del MNP y la oposición al uso del suplemento por parte del esposo. Los principales facilitadores fueron: recibir información del personal de salud sobre la mejora del niño, la percepción de la madre de que el niño mejora y testimonios positivos sobre el MNP de familiares o vecinas. Conclusiones. Existen barreras relacionadas al sistema de salud, al producto (MNP) y al comportamiento de niños, madres y familia / comunidad, que son necesarios abordar con estrategias que permitan superar estas dificultades, ya que impiden que los niños consuman diariamente, en una preparación adecuada el suplemento de MNP.
\end{abstract}

Palabras Clave: Anemia, Niños, Investigación cualitativa, Micronutrientes, Barreras de comunicación (fuente: DeCS BIREME)

\section{BARRIERS AND FACILITATORS OF MICRONUTRIENT POWDER SUPPLEMENTATION: MATERNAL PERCEPTIONS AND DYNAMICS OF HEALTH SERVICES}

\begin{abstract}
Objectives. To explore the barriers and facilitators of micronutrient powder (MNP) supplementation to mothers of children aged 6 to 35 months living in the Peruvian cities of Tacna, Loreto, Puno, and Lima. Materials and methods. This qualitative exploratory study with a grounded theory approach was conducted in the Peruvian cities of Lima, Tacna, Loreto, and Puno in 2014. In-depth interviews were conducted with mothers of children younger than 3 years, and observations in health care facilities (HCFs) were made to determine the barriers and facilitators of MNP supplementation. Results. The barriers identified at the health care level were limited access to HCFs, negative rumors regarding the MNP in the clinics, and poor care by health personnel. The barrier at the product (MNP) level was the lack of knowledge about the supplement, including taste and side effects. The barrier at the maternal level was lack of time, neglect, difficulties in preparing the MNP, and opposition to the use of the MNP by the husband. The main facilitators were receiving information from health personnel regarding child improvement, mother's perception of child's improvement, and positive testimonies about the effectiveness of the MNP from relatives and neighbors. Conclusions. Barriers regarding MNP use at the health care system level and those associated with the behavior mothers and family/community, which prevent children from consuming the MNP supplement daily, need to be addressed through the development of strategies. Strategies must be developed to overcome the barriers related to the health care system, the MNP, and the behavior of children, mothers, and family/community because these barriers prevent children from consuming the MNP daily.
\end{abstract}

Key Words: Anemia, Children, Qualitative research, Micronutrients, Communication barriers (source: MeSH NLM).

\footnotetext{
Centro Nacional de Alimentación y Nutrición, Instituto Nacional de Salud. Lima, Perú.

Nutricionista, ${ }^{\mathrm{b}}$ Sociólogo

Recibido: 09/10/2017 Aprobado: 30/11/2017 En línea: 07/12/2016
}

Citar como: Aparco JP, Huamán-Espino L. Barreras y facilitadores a la suplementación con micronutrientes en polvo. Percepciones maternas y dinámica de los servicios de salud. Rev Peru Med Exp Salud Publica. 2017;34(4):590-600. doi: 10.17843/rpmesp.2017.344.3164 


\section{INTRODUCCIÓN}

En el Perú, para el año 2016, el 43\% de niños menores de tres años tenía anemia, lo que equivale a 948000 niños ${ }^{(1)}$. Estas cifras evidencian que, a pesar de los esfuerzos del gobierno, la anemia continúa siendo un problema grave de salud pública. Para revertir la anemia, el gobierno implementó la suplementación con micronutrientes en polvo (MNP) ${ }^{(2)}$, ya que es recomendado por la Organización Mundial de Salud ${ }^{(3)}$ y cuenta con evidencia de eficacia ${ }^{(4,5)}$ y efectividad ${ }^{(6,7)}$ para reducir la anemia.

La suplementación con MNP busca prevenir la anemia desde los seis primeros meses de vida, brindando hierro para cubrir los altos requerimientos del niño. Los MNP contienen hierro, zinc, vitamina C, A y ácido fólico. Además, el hierro se encuentra encapsulado en una capa de lípidos, por lo que no tiene interacción con los alimentos, esta fortificación casera no altera el sabor de las comidas, y es de fácil y rápida preparación ${ }^{(8)}$. La norma vigente dispone que todo niño de seis meses que asiste a algún establecimiento de salud (ES) del Ministerio de Salud (MINSA) de Perú, debe recibir mensualmente 30 sobres de MNP durante 12 meses, para ser consumidos de manera diaria. Asimismo, para su uso se indica que el sobre de MNP se debe mezclar en dos cucharadas de comida de consistencia semisólida, a temperatura tibia y que, el consumo debe darse en los primeros 15 a $20 \mathrm{~min}$. Cuando no se cumplen estas condiciones la capa lipídica que cubre el hierro del MNP puede romperse, exponiendo el hierro al oxígeno y ocasionando cambios en el color, sabor e incluso olor de la comida ${ }^{(9)}$.

A pesar de que existe evidencia de la eficacia de los MNP a nivel mundial $(4,5,10,11)$, en Perú la intervención basada principalmente en el suplemento de MNP no ha reducido la anemia (1). Esta falta de impacto se explicaría por las fallas de implementación que se han reportado (12-14). Otros países también encontraron barreras a la suplementación con MNP y dado que el objetivo biológico de aumentar la hemoglobina estaba mediado por los servicios de salud, por el cuidador (madre, familiar, etc.) y por el producto; estudiaron estos tres procesos como las vías de impacto del programa y como factores que ocasionan fallas a la implementación del mismo ${ }^{(7,15,16)}$.

Las intervenciones socialmente complejas, que involucran múltiples actores con diversos roles y con una forma propia de entender y experimentar el proceso, como el caso de la suplementación con MNP; deben evaluarse en su contexto y con la finalidad de comprender el proceso ${ }^{(17)}$. Con ese objetivo se diseñó un estudio cualitativo para explorar las barreras y facilitadores a la suplementación con MNP en madres de niños de 6 a 35 meses de edad de las regiones de Tacna, Loreto, Puno y Lima en Perú.

\section{MENSAJES CLAVE}

Motivación para realizar el estudio. La suplementación con micronutrientes en polvo (MNP) en el Perú, ha mostrado fallas en la implementación, por lo que es necesario explorar las barreras a la intervención y diseñar estrategias que permitan mejorar su desempeño.

Principales hallazgos. Se encontraron barreras como dificultades para acceder al establecimiento de salud, el sabor, los efectos secundarios, los rumores negativos y las dificultades en la preparación del MNP; así como la falta de tiempo y el olvido de la madre.

Implicancias. Es necesario abordar las barreras a la suplementación con MNP, diseñando estrategias que permitan superar estos obstáculos y brindar orientación con pertinencia cultural para el uso y consumo de MNP.

\section{MATERIALES Y MÉTODOS}

\section{DISEÑO Y ÁREA DE ESTUDIO}

Estudio cualitativo, exploratorio basado en los principios y procedimientos de la teoría fundamentada ${ }^{(16)}$, que proviene del interaccionismo simbólico, cuya premisa es que las personas actúan respecto a las cosas, de acuerdo a los significados que estas tienen para ellos, estos significados surgen de la interacción social y existe un proceso interpretativo continuo ${ }^{(18)}$. Esto nos permitió acceder a los valores, ideas y prácticas con respecto al MNP. El estudio se desarrolló entre septiembre y noviembre de 2014, en el marco del Ensayo Comunitario para mejorar la adherencia a la suplementación con MNP y se realizó en madres de niños menores de tres años que acudían a ES del MINSA de las cuatro regiones con mayor prevalencia de anemia, en la costa se incluyeron los ES de la Dirección de Salud Lima Este y la Dirección Regional de Salud (Diresa) de Tacna, en la sierra se eligió a la Diresa de Puno y en la selva se eligió a la Diresa de Loreto.

\section{POBLACIÓN DE ESTUDIO}

Los sujetos de estudio fueron mujeres con hijos de entre seis y 35 meses de edad, que recibieron atenciones de salud en ES del MINSA y no se consideró indispensable que hayan recibido el suplemento de MNP. Usamos el muestreo teórico ${ }^{(19)}$ tomando en cuenta las dimensiones de interés. En cada zona no se consideraron subgrupos étnicos o lingüísticos. El proceso respondió al principio de saturación, a través del cual la recolección de datos cualitativos procedía hasta llegar al punto en que la información se repetía sistemáticamente.

\section{ENTREVISTAS}

Para el desarrollo del estudio cualitativo, se capacitaron antropólogos en la aplicación de la entrevista 
semiestructurada, en la obtención del consentimiento informado y en las pautas de observación. Las entrevistas se aplicaron a madres de niños menores de tres años en el ES o en sus hogares y tuvieron una duración promedio de 73 minutos. Los ejes temáticos o dimensiones se definieron en función de la dinámica de la suplementación con MNP y los antecedentes abordados por estudios previos $(7,12,15,16)$. los cuales fueron: a) conocimientos sobre MNP, b) atención en suplementación con MNP, c) barreras y facilitadores para la suplementación con MNP, d) Beneficios percibidos del MNPy d) recomendaciones para mejorar la suplementación con MNP. El instrumento se aplicó en pilotos en las cuatro regiones para adecuar las preguntas al contexto.

\section{PROCEDIMIENTOS}

El trabajo de campo se realizó entre los meses de agosto y octubre de 2014. Durantela recolección de datos participaron ocho antropólogos que efectuaron 212 entrevistas en las cuatro regiones, el equipo de investigadores realizó un monitoreo en campo, del avance del estudio y de las técnicas aplicadas. Además, se obtuvieron datos en la sombra, a través de la observación de la dinámica en los consultorios de Crecimiento y Desarrollo (CRED) e información con entrevistas informales al personal de salud que brindaba los MNP.

\section{ANÁLISIS DE DATOS}

Las grabaciones de las entrevistas se transcribieron en textos que fueron codificados para guardar la confidencialidad de los participantes. Los criterios de validez del estudio fueron credibilidad, auditabilidad y transferibilidad; para ello, dos investigadores realizaron la codificación de textos de manera paralela e independiente (credibilidad), la auditabilidad se garantiza con la disponibilidad de los audios, transcripciones y notas de campo del estudio y para la transferibilidad de resultados a contextos similares se describen las zonas de estudio y características de los participantes. La codificación se realizó en tres etapas: a) codificación abierta para identificar los conceptos básicos en los discursos; b) codificación axial que desarrolla dimensiones, categorías y subcategorías y c) codificación selectiva que permite integrar las categorías para organizarlas alrededor de un concepto explicativo central ${ }^{(19,20)}$. El proceso de análisis se realizó siguiendo la metodología de la teoría fundamentada y para asegurar la consistencia interna se aplicó el principio de comparación constante ${ }^{(18)}$. Todos los procedimientos se realizaron en el Software Atlas-Ti V6.

\section{ASPECTOS ÉTICOS}

El protocolo de estudio fue aprobado por el Comité de Ética del Instituto Nacional de Salud de Perú. Se solicitó a cada madre su consentimiento informado verbal para realizar y grabar las entrevistas.

\section{RESULTADOS}

Las participantes del estudio fueron 212 madres de entre 18 y 42 años, en la Tabla 1 se describen sus características.

\section{BARRERAS PARA LA ADHERENCIA A LA SUPLEMENTACIÓN CON MNP}

Los elementos emergentes en las entrevistas revelaron varios tipos de barreras, las que se organizaron en tres dimensiones que se muestran en la figura 1: barreras ligadas al sistema de salud, barreras ligadas al producto MNP y barreras ligadas a la madre/familia.

\section{BARRERAS LIGADAS AL SISTEMA DE SALUD}

Las barreras inherentes al sistema de salud se dividen en dos grandes categorías: barreras para obtener una atención en el ES y barreras durante el proceso de recibir los MNP (Tabla 2).

\section{Barreras para obtener una atención en el ES}

Las madres indican dificultades para acceder al ES, ya que el suplemento se recogía (o se obtenía la orden) en el consultorio de CRED, uno de los servicios con mayor demanda, que establece un número fijo de niños (cupo) para atención en el día. Otras madres refieren dificultades de acceso en niños mayores de un año, porque no cuentan con cita mensual para CRED, y por esto no eran recibidas por el personal de admisión o triaje. Las madres también revelan que en ocasiones asistían al ES por el MNP, pero

Tabla 1. Características de las madres de niños menores de 3 años.

\begin{tabular}{|c|c|}
\hline Característica & $\mathrm{n}=\mathbf{2 1 2}$ \\
\hline Media de la edad ( \pm DE) (años) & $28( \pm 1,3)$ \\
\hline \multicolumn{2}{|l|}{ Nivel educativo (\%) } \\
\hline Primaria o menos & $46(21,7)$ \\
\hline Secundaria & $110(51,9)$ \\
\hline Superior & $56(26,4)$ \\
\hline \multicolumn{2}{|l|}{ Número de hijos (\%) } \\
\hline 1 & $101(47,6)$ \\
\hline 2 & $68(32,1)$ \\
\hline Más de 2 & $43(20,3)$ \\
\hline \multicolumn{2}{|l|}{ Situación laboral (\%) } \\
\hline Trabaja & $93(43,9)$ \\
\hline No trabaja & $119(56,1)$ \\
\hline \multicolumn{2}{|l|}{ Región (\%) } \\
\hline Lima Este & $67(31,6)$ \\
\hline Loreto & $25(11,8)$ \\
\hline Puno & $58(27,4)$ \\
\hline Tacna & $62(29,2)$ \\
\hline
\end{tabular}

DE: desviación estándar 
Tabla 2. Barreras percibidas por las madres, ligadas al establecimiento de salud.

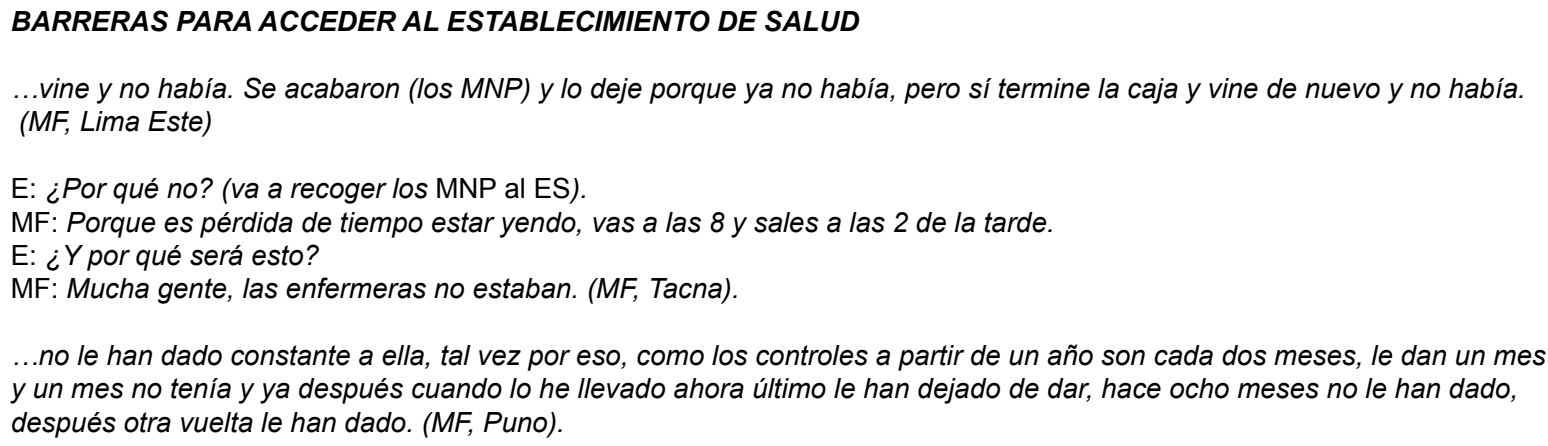

\title{
BARRERAS EN EL PROCESO DE RECEPCIÓN DEL MICRONUTRIENTE EN POLVO
}

\author{
... en el hospital (ES) escuche que las chispitas atrasaban a los niños, que hace que los niños no piensen, así se quejaban. \\ (MF, Puno)
}

La primera vez que vine (a recoger el MNP) la señora me contó (en la sala de espera) que no es buena esa chispita, que cosa todavía tendrá... su sobrino comió esa chispita y se puso todo "opa" (sonso). (MF, Tacna)

Aquí en la posta es donde escuché hablar de las chispitas... varias (madres) me dijeron que le cayó mal a su hijo, que le quitaba el apetito (al niño). (MF, Lima)

Yo llevaba a la posta porque no hacía caso, decía es mentira cómo va a ser así... pero después veía en la posta que no trataban bien, cuando decías algo te gritaban, por qué no atiendes en tu casa. Renegaba, si no quieren trabajar, mejor que se vayan, eso decía por dentro... empecé a desconfiar de los doctores porque no trataban bien. (MF, Puno)

Una vez me dieron a los siete meses y ahora, tienen un año y es que no he venido a la posta como tres veces. Me dieron para un mes. (MF, Lima)

...no le di porque me dieron vencido. No le di, pero la señorita me dijo: ...tiene todavía tres meses válidos. Entonces uno por evitar cualquier cosa que pueda pasar, no le di. (MF, Puno)

...En la clínica me dijeron que no (no dar la chispita) porque le puede hacer daño a la bebe. (MF, Lima)

Tendría que decirme un médico de qué está hecho y por qué me están dando, claro, me está dando una enfermera en una posta, pero realmente no me dice un médico, me gustaría que me recete un médico qué vitaminas tengo que darle. (MF, Tacna)

ES: establecimiento de salud; MNP: micronutriente en polvo; MF: madre de familia

no las atendían, porque no se encontraba el personal encargado de brindar la suplementación (Tabla 1).

\section{Barreras en el proceso de recepción de MNP}

Una vez que la madre logra acceder a un turno de atención, operan nuevas barreras dentro del ES, varias madres refieren que mientras se encontraban en la sala de espera, otras madres les comentaban aspectos negativos del MNP (Tabla 2). Otro aspecto manifestado por las madres era el tiempo excesivo para recibir el suplemento, y que, al contar ya con la orden, no pudieron recoger el MNP; en algunos casos porque no había suplemento $\mathrm{y}$, en otros casos, porque el servicio de farmacia estaba cerrado.

Por otra parte, algunas madres, sobre todo de Loreto y Puno, revelaron que en algunas ocasiones les entregaron suplementos vencidos, por lo que empezaron a perder la confianza en el personal de salud. Las madres también indicaron que no recibían un buen trato del personal de salud, además, sienten que no se les da toda la información, que siempre las atienden rápido para acudir a realizar otra función o atender a otra persona. Finalmente, un aspecto particular para Lima y Tacna, surge de los testimonios de las madres sobre la confianza en el producto, pues manifiestan que no usan el MNP porque en las clínicas o consultorios particulares los médicos le dicen que no lo usen y dado que es recomendado solo por las enfermeras, deciden no usarlo (Tabla 2).

\section{BARRERAS LIGADAS AL PRODUCTO (MNP)}

Muchas de las madres muestran un limitado conocimiento sobre el MNP y expresan que no saben con precisión que contienen el sobrecito del MNP; algunas refieren que son 
vitaminas, sin embargo, la mayoría no reconoce o identifica al hierro como parte del suplemento, ni que está asociado a combatir la anemia. Otro aspecto a considerar es la forma de presentación del MNP, sobre todo en Puno y Tacna, donde destaca una percepción negativa a la presentación en polvo, pues se asocia con tierra, suciedad y arena, de tal forma que, a veces, las madres sienten que le agregan algo sucio a la comida del niño (Tabla 3 ).

Tabla 3. Barreras percibidas por las madres, ligadas al producto (micronutriente en polvo).

\section{CONOCIMIENTO DEL MICRONUTRIENTE EN POLVO}

... le das a tu hijito nomás, no me han dicho nada. (MF, Puno)

La verdad que también no sabemos qué contiene, para qué sirve (el MNP). (MF, Puno)

Bueno, le ayuda en su alimentación, tendrá, pues, vitaminas, no sé qué vitaminas tendrá, pero sé que le refuerza su alimentación me han dicho, pero no me han dado. (MF, Tacna)

Yo creo que es vitamina, son nutrientes para que no tenga su anemia. (MF, Loreto)

Es para que le complementemos con la comida, para que tengan una mejor absorción de vitaminas. (MF, Lima)

\section{FORMA DE PRESENTACIÓN}

...porque las chispitas son medio oscuritas y a veces dicen no, esto es sucio, es sucio, no la quiere, mami está sucia, está cochino, tierra hay y no quiere comer, yo pienso que si sería un jarabe se lo tomaría. (MF, Puno)

... esos puntitos negritos que salen porque a veces los niños lo ven y dicen, cochino. Eso es lo que dice ella, porque la primera vez que se lo di, se lo di en leche, luego me dijo la señorita que no debía darle ahí sino en comida, pero yo le di como sé que le gusta la leche y ella vio el biberón y vio la parte de abajito negrito y me dijo, cochino. (MF, Tacna)

Su color, que sea blanquito, porque es medio negrito, arena parece. Porque a veces mi hijita, a pesar de que yo lo escondo, mira como diciendo qué es, porque eso es como arenita. (MF, Lima)

\section{SABOR}

No sé, cómo sentirá. Yo pienso que siente así como un sabor diferente, así como por ejemplo cuando yo como, le echo Ajinomoto y le siento un sabor diferente, yo siento que ella también podría sentir. (MF, Tacna)

Tenía mucho hierro creo, tenía sabor a lata. (MF, Tacna)

Se quejaban, solo decía pica, pica. (MF, Puno)

Su sabor tiene rico oloroso tiene, dice. Por eso le come el muchacho, dice. (MF, Loreto)

Es un poco medio suero, sí lo cambiaría que sea un poco dulcecito. (MF, Lima)

\section{MEDICAMENTO}

Creo que mejor es darle al natural, una ensalada de betarraga que unas chispitas. Cómo estarán, de cuánto tiempo estarán ahí... De repente, tiene otro efecto... (MF, Tacna)

Prefiero lo natural porque no tiene consecuencias. Porque a veces algunos recetaban unas pastillas para algo y después le duele el estómago. Tiene su contra, tiene su contra. No sabemos tampoco cómo lo habrán elaborado. En cambio, lo natural sabemos bien que son buenos esos alimentos y no le hacen nada. (MF, Tacna)

\section{EFECTOS NO ESPERADOS}

Le hace estreñir, está estreñida, por eso es que está tomando agüitas, así. He visto que se ha estado estriñendo. (MF, Puno)

... le ha hecho soltar su estómago la última vez que le di... y no le pasaba con nada. (MF, Puno)

Es cuando se indigestó, le di por dos días consecuentes, el primer día normal, no más le dio diarrea, el segundo día le volví a dar y le dio vómito y diarrea ya, de allí le quité y se alivió. (MF, Loreto)

A la semana se comenzó a estreñir, luego esta semana que no le he dado, no se ha vuelto a estreñir. Mientras que le daba las chispitas le daba lactulosa para que se desestriña. (MF, Lima)

...A algunos no les cae bien y les estriñe y a algunos lo contrario. (MF, Lima)

La primera vez le di y se subió de peso, el mes pasado no le di...porque subió un poco de peso, porque la idea es que no se engorde mucho, ahora este mes me han dicho que se lo vuelva a dar por los nutrientes, que es importante, pero la idea es que se mantenga. (MF, Tacna)

ES: establecimiento de salud; MNP: micronutriente en polvo; MF: madre de familia 
En algunas zonas, el sabor también se percibía como una barrera, sin embargo, las opiniones eran diversas. En Tacna y Lima la mayoría de madres manifestaron que no tenía sabor, mientras que en Puno las madres refieren que el MNP tiene diversos sabores, desde amargo, agrio, hasta no tener un buen sabor; en tanto, en Loreto, las madres, sobre todo del área rural, mencionaban que el MNP tenía buen olor y un sabor agradable.

Algunas madres también refieren que al darles MNP a los niños notaban que sufrían de estreñimiento, deposiciones sueltas o no querían consumir la comida con el MNP, por lo que optaban por no darle el suplemento. Otra percepción fue que el MNP podría producirle algún daño al niño, las madres refieren que sus esposos y la comunidad les decían que ese producto era para que los niños sean calladitos (sumisos), para que no puedan tener descendencia, que se regalan solo a los pobres o lo comparaban con los casos de intoxicación que alguna vez ocurrieron en el programa de alimentación escolar Qaly Warma.

\section{BARRERAS LIGADAS AL COMPORTAMIENTO DE LAS MADRES, NIÑOS Y COMUNIDAD FRENTE AL MNP}

\section{Madres}

En sus discursos las madres revelan las dificultades para recibir los MNP (Tabla 4). En las zonas urbanas muchas madres refieren que no tienen tiempo para asistir al ES a recoger los MNP, porque estudian o trabajan y consideran que el tiempo a invertir para el recojo es excesivo. Mientras que en el ámbito rural algunas madres no acuden al ES porque están en temporada de siembra o no tienen los recursos para movilizarse. Otra barrera importante es que las madres no tienen tiempo para darle el MNP al niño, pues requieren de 30 a 45 min para darle la comida. Además, otras madres indican que se olvidan de dar el MNP al niño, porque tienen que hacer múltiples actividades, tanto en el hogar como en el trabajo. En muchos casos, las madres tienen la idea de que solo se da en el almuerzo, por lo que al recordar que no le dieron el MNP, esperan al día siguiente.

Muchas madres refieren imprecisiones y confusión sobre cómo preparar el MNP. Una de las indicaciones que trae más problemas es la cantidad de comida en la que hay que preparar el MNP, algunas refieren que es en dos cucharaditas, otras en cinco cucharadas, en medio plato o en todo el plato. Asimismo, la consistencia del alimento también es otro aspecto problemático, pues algunas madres refieren haber recibido la indicación de prepararlo en líquidos. También hay madres que, a pesar de conocer bien las indicaciones sobre la preparación, lo realizan de forma diferente, pues manifiestan que no se puede usar como lo indican en el ES (Tabla 4).
Niños

Las barreras al consumo de MNP percibidas por las madres con respecto al niño son varias, muchas indican que los niños sienten el sabor y olor de los MNP cuando lo mezclan con su comida y que, al percibir estas características, dejan de comer. Otras madres refieren que los niños más pequeños de (entre 6 a 15 meses) consumen bien el MNP, mientras que los niños mayores, cuando observan que la madre prepara la comida añadiéndole el MNP, no lo quieren comer, por lo que las madres optan por esconderse para preparar el MNP.

En esta dinámica de suplementación con MNP encontramos hasta cuatro tipos de niño: a) niños que consumen el MNP de forma irregular (no diariamente); b) niños que inicialmente tuvieron dificultades y que a insistencia de la madre consumen MNP regularmente; c) niños que consumen regularmente el MNP y nunca han tenido dificultades, y d) niños que no consumen el MNP, porque ante alguna dificultad la madre dejo de darle el suplemento.

\section{Familia / comunidad}

Algunas madres manifiestan una fuerte oposición de parte del esposo a que el niño reciba el MNP, porque tienen desconfianza de los productos gratuitos que el gobierno entrega. Ellos alegan que se quiere someter a las poblaciones pobres con alimentos o suplementos que puedan volver a los niños más brutos, más sumisos, para que no crezcan e, incluso, que se vuelvan infértiles. En Puno, destacaba la analogía entre el MNP y los alimentos de Qaly Warma, que habían producido algunas intoxicaciones, pues ambos provenían del gobierno. De la misma forma, en la familia, algunas madres refieren que su propia mamá o suegra (abuelas del niño), hermanas o cuñadas les referían que no les den el MNP, porque criaron bien a sus hijos sin el suplemento y que los niños no lo necesitan; algunas incluso indicaban que los niños podían volverlo "soncito" o provocarle algún daño (Tabla 4).

\section{FACILITADORES A LA SUPLEMENTACIÓN CON MICRONUTRIENTES}

\section{FACILITADORES LIGADOS AL SISTEMA DE SALUD}

Las madres que mantenían una suplementación regular con el MNP, refieren que uno de los aspectos que más las motivaba, era que el ES les informaba del progreso del niño a través de los resultados de hemoglobina o verificaban las mejoras en el desarrollo motor, habla, progresos en caminar, etc. Otras madres destacan como un facilitador una adecuada consejería sobre el uso del MNP; mientras que otras madres también resaltaban los ES con horarios alternativos (tardes) para distribuir el MNP (Tabla 5). 
Tabla 4. Barreras ligadas al comportamiento de la madre, el niño y la comunidad frente al micronutriente en polvo

\section{MADRES}

\section{Dificultades para recibir los MNP}

Cuando empezó a comer sus chispitas no he podido ir a la posta por lluvia y granizo casi tres meses... (MF, Puno).

Desde hace 2 meses me han dado. Como no tenía tiempo para llevarla... y como demora la posta, no me alcanza el tiempo tampoco, así que no la he llevado. (MF, Tacna)

\section{Olvido de la madre}

Sí, pero a veces me olvido, hay veces que no estoy, no llevo (al ES). (MF, Lima)

Por cosas del día me olvidaba, yo pensaba que se daba en la mañana nomas, por eso a veces me olvidaba y ya no le daba más tarde, pero no era así se le podía dar a cualquier hora del día. (MF, Puno)

\section{Dificultades en la preparación del micronutriente en polvo}

Le saco un poquito de sopita ahí le mezclo y ahí le doy todos los días y a veces, me olvido. (MF, Lima)

Complicado no puede decir, porque no son complicadas, solo que a veces por cuestiones de tiempo ya no puedo darle. (MF, Tacna) Tienes que preparar una cucharada en su sopita, ahí hay que preparar (el MNP). (MF, Puno)

...abres el sobre... no lo vas a dar solo sus chispitas, en un quakercito ahí me ha dicho. (MF, Puno)

Hasta se lo doy cuando toma su matecito, ahí mismo le doy a veces, normal, porque no tienen ningún sabor, hace que el bebé normal lo pueda tomar. (MF, Tacna)

Sí, hay que...en un plato preparar todito nomás. (MF, Puno)

Primero como no sabía cómo darle le daba en la cucharita, ya luego le espolvoreaba nomás en su plato, pero siempre deja un poco (MF, Tacna).

Primero preparo la papilla y ahí le hecho, en 5 cucharaditas, ahi le hecho todo el sobrecito... (MF, Tacna)

El doctor dice en una cucharita agarras del plato y le metes. (MF, Loreto)

En una papilla, un par de cucharitas. Mezclarlo en su papilla el sobre y darle de comer. (MF, Lima)

Le doy lo más que puedo, es que por ejemplo con el tallarín no le puedo dar porque es seco, le daré más que nada con los estofados, con las lentejas, con el poroto. (MF, Tacna)

Hay alguna dificultad a veces por motivo de viajes no lo llevamos, pero cuando estamos en la casa le damos. Cuando a veces estamos en la casa ahí sí... No pues cuando lo lleva le da sol y se malogra. (MF,Loreto)

Si le da con arroz. Tres de estas bolsitas al día... En la mañana, tarde y noche. (MF, Loreto)

NIÑOS

Sí, pero se lo doy escondido (para que no vea el MNP), come todos los días. (MF,Lima)

El niño cuando es grandecito si ya sabe, ya no le gusta ya. Cuando está chiquito pues el sí puede comer muy bien... los muchachos no los quieren pues, porque tiene malos, como un olor... (MF, Loreto)

\section{FAMILIA / COMUNIDAD}

... mi esposo decía: no, no le vas a dar las chispitas, cómprale eso mejor... y ya no le he dado. Decía: no, del gobierno siempre viene con algo, o sino mira, ahí lo del Qali Warma, es del Gobierno, ¿cuántos niños se han muerto, se han intoxicado? ¿Eso quieres con ella? (MF, Puno)

A veces las señoras dicen que lo están mandando de otro país para que ellos se hagan más retrasaditos. (MF, Puno) Una hermana, ella también ha recibido, ella me dijo que le había dicho que no le dieran porque dice que hay una señora que le daba a su hijito y se ha vuelto sonsito. Algo así le ha dicho, no sé por qué. No sé si será bueno o malo, eso también quería preguntarle al pediatra, pero nunca le pregunté. (MF, Tacna)

...mi cuñada también me decía no le des, acaso mis hijos han comido, no le he dado. Más su comida le doy, el huevo, la carne. (MF, Tacna)

ES: establecimiento de salud; MNP: micronutriente en polvo; MF: madre de familia 
Tabla 5. Facilitadores de la suplementación con micronutrientes en polvo

\section{SISTEMA DE SALUD}

Sí, se ha recuperado, la doctora le ha dicho que se ha recuperado. (MF, Puno)

...me gustaba porque le subía el peso y le hicieron de nuevo eso de la anemia y salió un poco más, y le ha subido. (MF, Puno)

Subió de peso, de talla también, pero siempre ha estado bien de talla y peso. (MF, Tacna)

Ya está un poquito gordito dice (el personal de salud). Cuando estaba bien flaquito de ahí le da en su comida le come y ya de ahí esta gordito. Cuando trae a control ya sube ya. (MF, Loreto)

Hasta ahora le sigo dando y la doctora me dijo que está subiendo de peso. Me dieron esto para que pueda tener las vitaminas $C$ y todas las vitaminas. (MF,Lima)

Bien. Me gustó que sí subió de hemoglobina. En ese tiempo mi hija tenía 10,2 y después tenía casi 11,5 ya estaba bien... (MF, Lima)

Así como una persona que no sepa leer, normal, bien te decían: mira asi es la papilla, en esta cucharita le preparas con un poquito de agüita hervida, le preparas y le das. (MF, Puno)

Primero, dice el doctor que cocine primero aunque sea arrocito o tacachito, echar una de esa bolsitas y primero darle de comer poquito primero, después ya se da de comer lo que quiere. (MF, Loreto)

\section{PRODUCTO}

...se puede engañar, el sulfato ferroso el niño no tolera, entonces en esto se puede engañar porque como él tiene hambre, lo va a comer. (MF, Tacna)

Me han dicho que sí funcionan, ya no tienes que engañar diciendo que es jarabe, le echas a la comida no más y normal lo comen los niñitos, me han dicho. (MF, Tacna)

La vitamina, como es polvo se puede "esconder" en la comida y rápido lo comen los chicos...además no "pinta" su diente... (MF, Loreto)

Anteriormente se tomaba el sulfato ferroso en jarabe. Eso no lo tomaban mis hijos, en cambio el polvito sí. Antes no porque tenía un olor, tenía más ferroso era fuerte por eso no le tomaba. (MF, Lima)

\section{MADRE}

Porque ya le veo más, porque más moribundita era mi nena, más sonsita, tontita era y ahora más viva está. (MF, Puno) Está recuperando el peso, porque como le digo estaba así mal, estaba con fiebre y diarrea pero ahora le estoy viendo que está recuperando. (MF, Loreto)

Todo normal porque lo veo más hábil será que le está ayudando en su cerebrito, agarra su juguete. Antes era un poquito sumiso, miedoso, pero ahora no, cualquiera cosa patea, es una de sus defensas sus piernas y sus huesitos. Es fuerte, sus musculitos. Siento sus cachetadas que tiene más fuerza. Sí funciona. (MF, Lima)

Las chispitas (mi familia) me han dicho a mí que le abren apetito. (MF, Puno)

Cuando da al muchacho va a crecer mejor, así contaba la vecina. Anteriormente se daba jarabe para muchachos eso le daba. (MF, Loreto)

Mi cuñada me contó que su hijo se sano de la anemia solo comiendo esas chispitas. (MF, Lima)

ES: establecimiento de salud; MNP: micronutriente en polvo; MF: madre de familia

\section{FACILITADORES LIGADOS AL MNP (PRODUCTO)}

Respecto a las características del producto que facilitan su uso, la Tabla 5 muestra que algunas madres, al comparar el sulfato ferroso con el MNP, refieren que este último es mejor porque se puede esconder en la comida y que si lo preparan bien, el niño no sentirá el sabor del suplemento. Otro aspecto que destacan, es que con el MNP saben, con seguridad, la cantidad exacta de suplemento a dar al niño. Asimismo, señalan que el MNP no tiene efectos desagradables como pintar los dientes. 


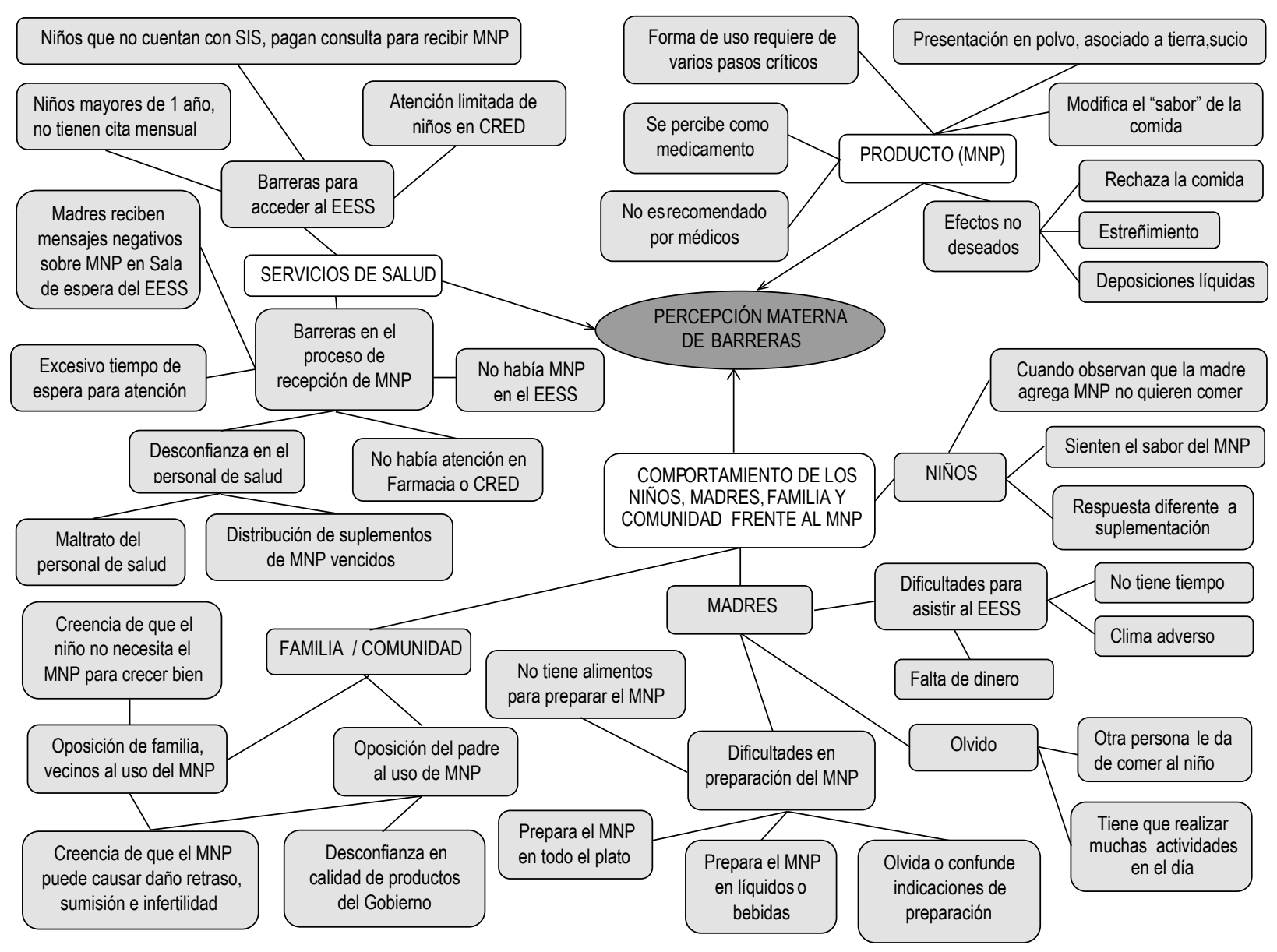

Figura 1. Barreras a la suplementación con micronutrientes en polvo a nivel de servicios de salud, producto y comportamiento de niños, madres y familia / comunidad

\section{FACILITADORES LIGADOS A LA MADRE}

Las madres revelan que cuando ellas perciben mejoras en sus hijos, también se motivan a seguir dándoles el MNP, ellas ven las mejoras en el apetito, lo ven más activo, con mejor color, incluso algunas señalan, que ya sabían que no tenía anemia, antes de tener los resultados de hemoglobina en el ES (Tabla 5). Otras madres revelan que dan el MNP a sus hijos regularmente, a pesar de las dificultades, porque están muy motivadas a asegurar el ideal crecimiento de sus hijos y que puedan ser niños sanos, inteligentes y felices. La motivación la toman de diferentes fuentes, a veces son ellas mismas las que se automotivan, en otros casos se sensibilizaron con la consejería en el ES y otras veces, refieren que alguna amiga, vecina o familiar le dio su testimonio positivo sobre el uso de MNP y el progreso significativo de su hijo.

\section{DISCUSIÓN}

Los hallazgos del estudio evidencian barreras para la implementación efectiva de la intervención con MNP y que es necesario abordarlas con estrategias que permitan superar estas dificultades que impiden que los niños consuman el MNP. Tanto las barreras identificadas, como los facilitadores, son insumos importantes para elaborar estrategias comunicacionales con pertinencia cultural, que motiven el uso apropiado y consumo de MNP.

Entre las barreras identificadas destacan las referidas al ES, otros estudios también han mostrado barreras para acceder al ES y, por ende, al MNP. Huamán-Espino et al. reportaron que en Apurímac el $22 \%$ de niños recibió menos de 60 sobres de MNP ${ }^{(14)}$. Otro estudio en Huanta da cuenta que un $21 \%$ de niños no recibió MNP de forma continua, ya que la madre no acudió al ES ${ }^{(13)}$. Una revisión sistemática de estudios cualitativos sobre barreras al acceso a la atención de salud, encontró hasta 230 tipos de barreras, siendo el $38 \%$ referidas al aspecto de aceptabilidad, $29 \%$ a accesibilidad, $22 \%$ a contacto y $11 \%$ a disponibilidad; estos hallazgos, de manera similar a nuestro estudio, reflejan la inequidad social (21).

El estudio también encontró que la orientación del personal de salud para el uso y consumo de MNP es importante, 
aunque no es la adecuada, resultados similares encontraron Creed et al. ${ }^{(12)}$. Asimismo, un estudio en Kenia, encontró buena aceptabilidad del MNP y evidenció como barreras potenciales a la falta de conocimiento y experiencia con el MNP, a la accesibilidad y al costo del suplemento ${ }^{(22)}$.

Respecto a las barreras referidas al producto, algunos estudios han mostrado que los suplementos de MNP pueden presentar resistencias iniciales a su consumo, si es que no existe una estrategia de mercadeo social, que comunique de forma clara y sencilla, que es el suplemento, como se usa y sus beneficios ${ }^{(22-24)}$. Otras barreras relacionadas al MNP, como la percepción de que es un medicamento, sabor desagradable, efectos secundarios o la asociación a tierra, no se han reportado en otros estudios de Perú ${ }^{(12-14)}$. Por su parte, las barreras referidas a la familia y comunidad, ejercen una gran influencia para la adopción de las recomendaciones de cómo usar el MNP, al respecto, un estudio en Perú y otro en México encontraron resultados similares para el uso de suplementos alimentarios, dando cuenta de la fuerte influencia de la familia y la comunidad ${ }^{(12,25)}$

El estudio también destaca que a nivel de sistema de salud, las motivaciones para el uso de MNP serían la retroalimentación del personal de salud sobre la evolución del niño, la orientación adecuada al contexto y el horario alternativo para recoger los MNP; estos hallazgos son similares a los de Creed et al. quienes también reportan otros facilitadores como las visitas domiciliarias, sesiones demostrativas y mezclar el MNP con plátano ${ }^{(12)}$, nosotros no encontramos referencia de estas estrategias, las cuales mejoran la pertinencia cultural de esta intervención tan compleja ${ }^{(26)}$. Las madres resaltan las ventajas del MNP sobre el sulfato ferroso, como que el suplemento se puede esconder en los alimentos, se siente menos el sabor y no tiñe los dientes; no encontramos estudios que hayan explorado este aspecto. Asimismo, se encontró que las madres también están muy motivadas a seguir la suplementación, cuando ellas mismas perciben las mejoras de sus niños y reciben el testimonio de algún familiar, igual que en dos estudios de Perú y Nigeria ${ }^{(12,27)}$.
El estudio tiene limitaciones inherentes al diseño de estudios cualitativos, como es la representatividad de las percepciones, dado que se realizó un muestreo teórico, que no permite inferencias. Una fortaleza del estudio, es su realización en cuatro regiones del Perú, lo que permite tener un panorama bastante amplio para explorar el uso y consumo en Perú, asimismo, si bien, no se pudo triangular la información con otras técnicas de recolección como grupos focales, las entrevistas se contrastaron con los datos en la sombra (observación directa) y datos provenientes del personal de salud, de cada una de las zonas de estudio ${ }^{(28)}$.

Los hallazgos del estudio indican que el éxito de una intervención contra la anemia, no radica solo en adquirir y entregar un producto eficaz, sino requiere, además, de un abordaje de las múltiples dimensiones de la adherencia terapéutica (29), más aun, cuando la intervención se distribuye, monitorea y evalúa a través del sistema de salud. Mención especial merece la escasa cobertura de los ES públicos que llegan al $30 \%$ de la población, lo cual limita el impacto en intervenciones basadas en ES ${ }^{(30)}$.

En conclusión, el estudio muestra que existen barreras a la suplementación con MNP en varios niveles, que impiden un adecuado uso y consumo del MNP, por tanto, es necesario abordar estas barreras para diseñar estrategias de promoción y educación con pertinencia cultural que permitan despejar las dudas y preocupaciones de la madre, la familia y la comunidad y deje en claro la importancia y los beneficios del consumo de MNP. Asimismo, es importante rescatar los facilitadores de la suplementación, para posicionar estrategias con el fin de construir mensajes adecuados a cada realidad.

Contribuciones de autoría: JPA y LHE han participado en la concepción del artículo, su redacción y aprobación de la versión final. Además JPA participó en la recolección de datos.

Fuente de financiamiento: Instituto Nacional de Salud.

Conflicto de interés: los autores no refieren conflictos de interés en la publicación de este artículo.

\section{REFERENCIAS BIBLIOGRÁFICAS}

1. Instituto Nacional de Estadística e Informática. Indicadores de Resultados de los Programas Presupuestales 2011-2016. Encuesta Demográfica y Salud Familiar (Resultados Preliminares) [Internet]. Lima: Instituto Nacional de Estadística e Informática; 2017. [citado 15 julio 2017] Disponible en: https://proyectos.inei.gob. pe/endes/images/Peru_Indicadores_de_ PPR_2011_2016.pdf

2. UNICEF. Sistematización de la experiencia peruana sobre suplementación con micronutrientes en los departamentos de: Apurímac, Ayacucho y Huancavelica 2009-2011. [Internet]. Lima: UNICEF, Ministerio de Salud, Programa Mundial de Salud; 2014. [citado 15 julio 2017]. Disponible en: https://www.unicef.org/ peru/spanish/SISTEMATIZACION_ PILOTO_Multimicronutrientes_ MINSA_UNICEF_PMA.pdf

3. Guideline: Use of multiple micronutrient powders for point-of-use fortification of foods consumed by infants and young children aged 6-23 months and children aged 2-12 years [Internet].
Geneva: World Health Organization; 2016. [citado 15 julio 2017]. Disponible en: http://apps.who.int/iris/bitstre am/10665/252540/1/9789241549943eng.pdf ?ua $=1$

4. Inayati DA, Scherbaum V, Purwestri RC, Wirawan NN, Suryantan J, Hartono $S$, et al. Combined intensive nutrition education and micronutrient powder supplementation improved nutritional status of mildly wasted children on Nias Island, Indonesia. Asia Pac J Clin Nutr. 2012;21(3):361-73. 
5. Sazawal S, Dhingra P, Dhingra U, Gupta $\mathrm{S}$, lyengar V, Menon VP, et al. Compliance with home-based fortification strategies for delivery of iron and zinc: its effect on haematological and growth markers among 6-24 months old children in north India. J Health Popul Nutr. 2014 Jun;32(2):217-26.

6. World Vision Mongolia. Report on the Effectiveness of Home-Based Fortification with Sprinkles in an Integrated Nutrition Program to Address Rickets and Anemia [Internet]. Ulaanbaatar: World Vision Mongolia; 2005. [citado 15 julio 2017]. Disponible en: http://www.wvi.org/ nutrition/publication/mongoliasprinkles-evaluation-report-2005

7. Loechl CU, Menon P, Arimond M, Ruel MT, Pelto G, Habicht JP, et al. Using programme theory to assess the feasibility of delivering micronutrient Sprinkles through a food-assisted maternal and child health and nutrition programme in rural Haiti. Matern Child Nutr. 2009;5(1):33-48. doi: 10.1111/j.17408709.2008.00154.x.

8. Zlotkin SH, Schauer C, Christofides A, Sharieff W, Tondeur MC, Hyder SMZ. Micronutrient sprinkles to control childhood anaemia. PLoS Med. 2005;2(1):e1.

9. Directiva Sanitaria para la prevención de anemia mediante la suplementación con micronutrientes y hierro en niñas y niños menores de 36 meses de edad. Resolución Ministerial $\mathrm{N}^{\circ}$ 055-2016. Ministerio de Salud, (12 de abril de 2017). Disponible en: ftp://ftp2.minsa.gob.pe/ normaslegales/2017/RM_250-2017MINSA.PDF

10. de Barros SF, Cardoso MA. Adherence to and acceptability of home fortification with vitamins and minerals in children aged 6 to 23 months: a systematic review. BMC Public Health. 2016;16:299. doi:10.1186/s12889 016-2978-0.

11.Salam RA, MacPhail C, Das JK, Bhutta ZA. Effectiveness of Micronutrient Powders (MNP) in women and children. BMC Public Health. 2013;13 Suppl 3:S22. doi: 10.1186/1471-2458-13-S3-S22.

12. Creed-Kanashiro H, Bartolini R, Abad M, Arevalo V.Promoting multi-micronutrient powders (MNP) in Peru: acceptance by caregivers and role of health personnel. Matern Child Nutr. 2016 Jan;12(1):15263. doi: $10.1111 / \mathrm{mcn} .12217$.

13. Fundación Acción contra el Hambre. Anemia por deficiencia de hierro y suplementación con multimicronutrientes en niños y niñas de 6 a 35 meses de edad. Situación en 4 distritos de la Provincia de Huanta, región Ayacucho [Internet]. Lima: Fundación Acción contra el Hambre;2012. pp 145. [citado 15 julio 2017]. Disponible en: http://intersocial. pe/wp-content/uploads/2014/10/pub1. pdf

14. Huamán-Espino L, Aparco JP, NuñezRobles E, Gonzáles E, Pillaca J, MaytaTristán P. Consumo de suplementos con multimicronutrientes Chispitas y anemia en niños de 6 a 35 meses: estudio transversal en el contexto de una intervención poblacional en Apurímac, Perú. Rev Peru Med Exp Salud Publica. 2012;29(3):314-23.

15. Kodish S, Rah JH, Kraemer K, de Pee S, Gittelsohn J. Understanding low usage of micronutrient powder in the Kakuma Refugee Camp, Kenya: findings from a qualitative study. Food Nutr Bull. 2011;32(3):292-303. doi: $10.1177 / 156482651103200315$.

16. Nguyen M, Poonawala A, Leyvraz M, Berger J, Schofield D, Nga TT, et al. A Delivery Model for Home Fortification of Complementary Foods with Micronutrient Powders: Innovation in the Context of Vietnamese Health System Strengthening. Nutrients. 2016;8(5). pii: E259. doi: $10.3390 /$ nu8050259.

17. Wolff N. Randomised trials of socially complex interventions: promise or peril? J Health Serv Res Policy. 2001;6(2):123-6.

18. Maxwell JA. Qualitative Research Design: An Interactive Approach. Ohio: SAGE Publications; 1996.

19. Strauss A, Corbin J. Bases de la investigación cualitativa: Técnicas y procedimientos para desarrollar la Teoría Fundamentada. Medellin: Universidad de Antioquia; 2002.

20. Glaser B, Strauss A. The discovery of grounded theory: Strategies for qualitative research. 8va ed. New York: Aldine Transaction; 1967.

21. Hirmas Adauy M, Poffald Angulo L, Jasmen Sepúlveda AM, Aguilera Sanhueza X, Delgado Becerra I, Vega Morales J. Barreras y facilitadores de acceso a la atención de salud: una revisión sistemática cualitativa. Rev Panam Salud Publica. 2013;33(3):223-9.

22. Jefferds ME, Ogange L, Owuor M, Cruz $\mathrm{K}$, Person B, Obure A, et al. Formative research exploring acceptability, utilization, and promotion in order to develop a micronutrient powder (Sprinkles) intervention among Luo families in western Kenya. Food Nutr Bull. 2010 Jun;31(2 Suppl):S179-85.

23. Griffiths, M. Communication strategies to optimize commitments and investments in iron programming. J. Nutr. 2002;132: 834S-838S.

24. Neufeld LM, Cameron BM. Identifying nutritional need for multiple micronutrient interventions. J Nutr. 2012;142(1):166S-72S. doi: 10.3945/ jn.111.138677.

25. Escalante-Izeta E, Bonvecchio A, Théodore F, Nava F, Villanueva MA, Rivera-Dommarco JA. Facilitadores y barreras para el consumo del complemento alimenticio del Programa Oportunidades. Salud Publica Mex. 2008;50(4):316-24.

26. Osei A, Septiari A, Suryantan J, HossainM, Chiwile F, Sari M, et al. Using formative research to inform the design of a home fortification with micronutrient powders (MNP) program in Aileu District, TimorLeste. Food Nutr Bull. 2014;35(1):68-82.

27. Tripp K, Perrine CG, De Campos P, Knieriemen M, Hartz R, Ali F, et al. Formative research for the development of a market-based home fortification programme for young children in Niger. Matern Child Nutr. 2011;7 Suppl 3:82-95. doi: 10.1111/j.1740-8709.2011.00352.x.

28. Flick U. The SAGE Handbook of Qualitative Data Analysis. London: SAGE Publications; 2014, pp. 125-143.

29. Organización Mundial de Salud. Adherencia a los tratamientos a largo plazo. Pruebas para la acción [Internet]. Ginebra: Organización Mundial de Salud; 2004. [citado 15 julio 2017]. Disponible en: http://www.farmacologia.hc.edu.uy/ images/WHO-Adherence-Long-TermTherapies-Spa-2003.pdf

30. Dirección General de Epidemiología. Análisis de la Demanda y el Acceso a los Servicios de Salud en el Perú. Lima: Dirección General de Epidemiología, Ministerio de Salud; 2012. [ciado 15 julio 2017]. Disponible en: http://www.dge. gob.pe/publicaciones/pub_asis/asis31.pdf

Correspondencia: Juan Pablo Aparco Balboa

Dirección: Jr. Tizón y Bueno 276 Jesús María. Lima, Perú

Teléfono: (+51) 989776969

Correo electrónico:jp_aparco@hotmail.com 